\title{
Development Dilemma and Its Solution of Economic Management National Experimental Teaching Demonstration Center
}

\author{
Sufeng Li, Xiaojing Chang, Teng Le, Chengxiang Hu, Shengli Wang, Wuqi Yang
}

Hebei GEO University, Shijiazhuang City, Hebei Province, China

Keywords: national experimental teaching demonstration center; predicament; management experimental teaching demonstration center; collaborative driving

\begin{abstract}
The development of experimental practice teaching has been greatly promoted through national experimental teaching demonstration center. With its increasing scale, the development dilemma, however, is gradually prominent. Focusing on the low cooperative level of the experimental teaching demonstration center, this paper puts forward the way to solve this predicament. Firstly, it enhances the level of cooperation between the experimental teaching demonstration center and the sibling department. Secondly, it strengthens the cooperation between experimental teaching demonstration center and the industry and enterprises. Finally, it builds a "virtual reality" platform for the sake of realizing the sustainable development of economic management of experimental teaching demonstration center.
\end{abstract}

\section{Preface}

With the rapid development of the economy, the society has continuously improved the specifications and standards of talent demand. The cultivation of practical talents has indicated the direction for the reform of university education. A lot of experimental practice teachings are carried out. It is a difficult problem to realize the maximization of the utility of the national-level economics experimental teaching demonstration center from the perspective of internal and external coordination. However, the consequent construction bottlenecks continue to be highlighted. The construction of the national experimental teaching demonstration center is characterized by insufficient professional features, heavy hardware upgrades and software upgrades, and rigid project funding systems, etc[1-2]. It becomes the key issue to how to make use of the benefits of the demonstration center and how to achieve sustainable development [3-4]. Meanwhile, the degree of experimental teaching is not enough, which hinders the development speed of national experimental teaching demonstration centers [5-6].So it requires to value the reform and construction of laboratories [7].

In summary, there is little research on the sustainable development of state-level experimental teaching demonstration centers from the perspective of internal and external collaborative innovation. This is exactly what makes this article unique.

\section{Dilemma faced by national experimental teaching demonstration center}

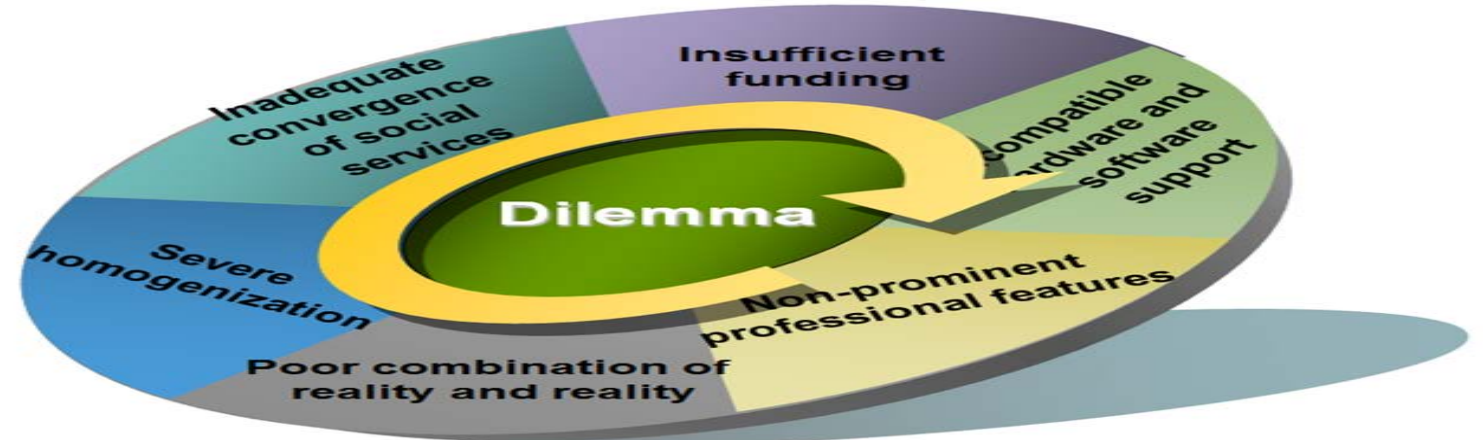

Figure 1 development dilemma faced by the national experimental teaching demonstration center 
The national-level economic management experimental teaching demonstration center not only provides a practical teaching place, but also undertakes the task of self-teaching. Though it has won a series of awards, it faces with many difficulties (fig 1).

\subsection{Insufficient investment in construction}

The engineering-based universities are not considered to be ample experimental construction funds. They meet the needs of science and engineering laboratory construction, and then meet the construction needs of other experimental teaching demonstration centers. As a professional laboratory of economic management, the investment in the hardware environment is relatively large while the investment in management software and software environment is relatively insufficient.

\subsection{Severe homogenization}

Many universities have imitated the construction of the center for experimental teaching and demonstration, but they did not give full play to the advantages of the school and neglected it. The laboratory built by the school is similar to the laboratory construction of ordinary institutions of higher learning in terms of form and content, but it has not yet truly reflected the school characteristics.

\subsection{Insufficient combination of virtual reality}

At present, the information teaching resources are mainly computer and course teaching software, but there are few professional comprehensive training platforms. The cross-professional comprehensive training platform is still in the initial stage of construction, which seriously inhibits interdisciplinary the ability to innovate. The video resources have not yet reached scale. Virtual simulation technology has not been fully applied in most universities. The demonstration center has greatly promoted the reform of experimental teaching. The radiation effect of the demonstration center, however, has had little effect. The combination of virtul reality has not been promoted yet.

\subsection{Unbalanced regional distribution}

The economic management experimental center is concentrated in the eastern regions while the midwestern institutions are relatively less. There are 50 economic management experimental centers, 30 of which are located in the east, accounting for $60 \%$ of the total, 12 of which are located in the west, accounting for $24 \%$ of the total, 8 of which are located in the central region, accounting for $16 \%$ of the total.

\subsection{Unsound external coordination mechanism}

There are serious "decoupling" between the school training and the actual needs of the industry in most universities. It has not yet formed a "one body and two wings" school-running mechanism, which has narrowed the employment exit of students. At the same time, the regional advantage has not yet been fully utilized in the management of experimental teaching. It is still not perfect for the collaborative mechanism of school-enterprise collaboration, which slows down the level of synergy between traditional experimental teaching and simulation experiment teaching.

\section{Ways to solve the dilemma: collaborative innovation driven}

\section{1 internal collaborative innovation driven}

\subsubsection{Integration of teaching, management and scientific research}

The goal of experimental practice teaching is to cultivate qualified comprehensive talents for society. Therefore, we should adhere all the training for the cultivation of talents. The experimental teaching demonstration center should be boldly transformed in order to realize the transformation from purely teaching to the integration of teaching, management and. It can be seen from this that a dynamic demand database for social talents has been established. It is a two-way driven to construct heoretical and practical teaching, which is high-quality talents with good learning abilities, high 
levels of practice and strong adaptability.

\subsubsection{Building a strong experimental teaching support system}

The experimental teaching demonstration center is an important bridge for the management of specialized teaching units. Horizontally, it has the functions of teaching software and hardware of the economic management experiment. In order to adapt to the rapid development of knowledge, it has established a long-term training mechanism and sent teachers to first-class universities to study regularly. Many comprehensive platforms were built (Fig 2).

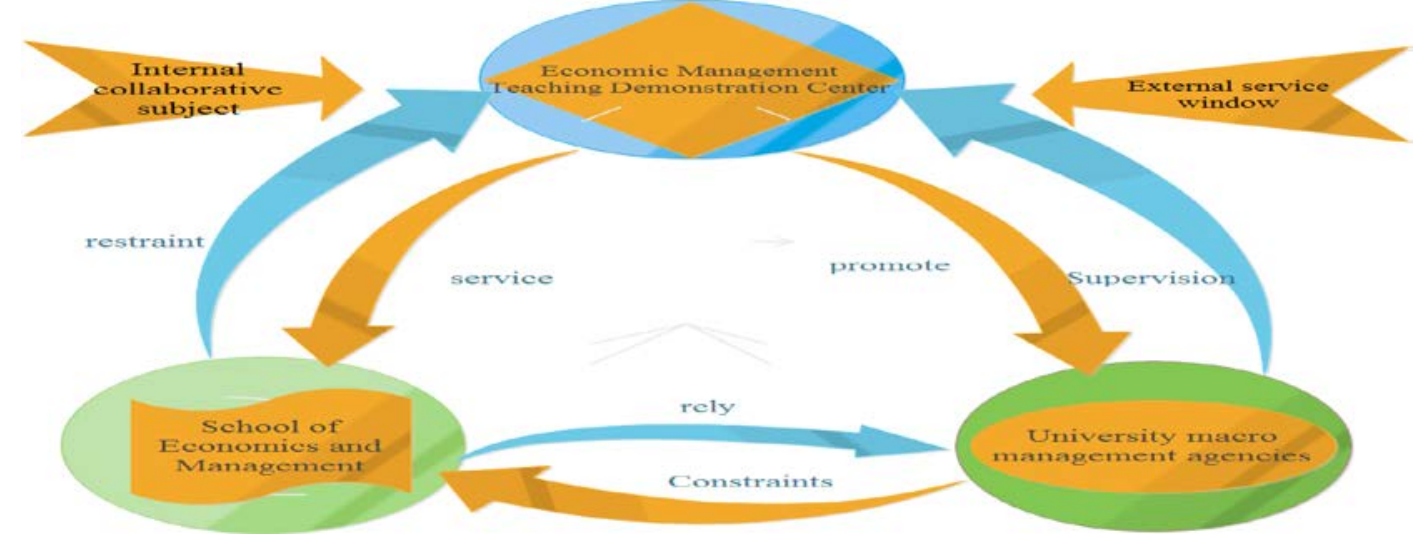

Figure 2 Internal collaborative driving of the national experimental teaching demonstration center

\subsection{Crack the dilemma: internal and external collaborative driven}

\subsubsection{Coordinating the relationship between demonstration center, company and the industry}

According to the characteristics of the university, the experimental teaching demonstration center manages students to ensure that they have a solid grasp of the knowledge, which can need the society for specialty professionals. It not only plays an experimental teaching assistant function, but also is responsible for liaising closely with the industry. Actively, we establish long-term cooperative relationships with social-related enterprises, which provides internship positions for students on a regular basis, and which can realize the integration of theoretical study and business management.

\subsubsection{Build an experimental training platform for combination of reality and practice}

The virtual experiment teaching demonstration center saves the huge cost of students' traditional social internship. It also finds out its own knowledge defect, meanwhile it inherits the basic principle of "combination of reality and practice". So the analysis shows that the sustainable development of the national experimental teaching demonstration center requires the mutual cooperation between the experimental teaching demonstration center and the industry and enterprises. It is also necessary to give full play to the virtual environment of modern experimental teaching, and also make up for the lack of traditional experimental teaching. At the same time, the virtual experimental teaching demonstration center fully depends on the disciplines of the traditional experimental teaching demonstration center(Fig 3).

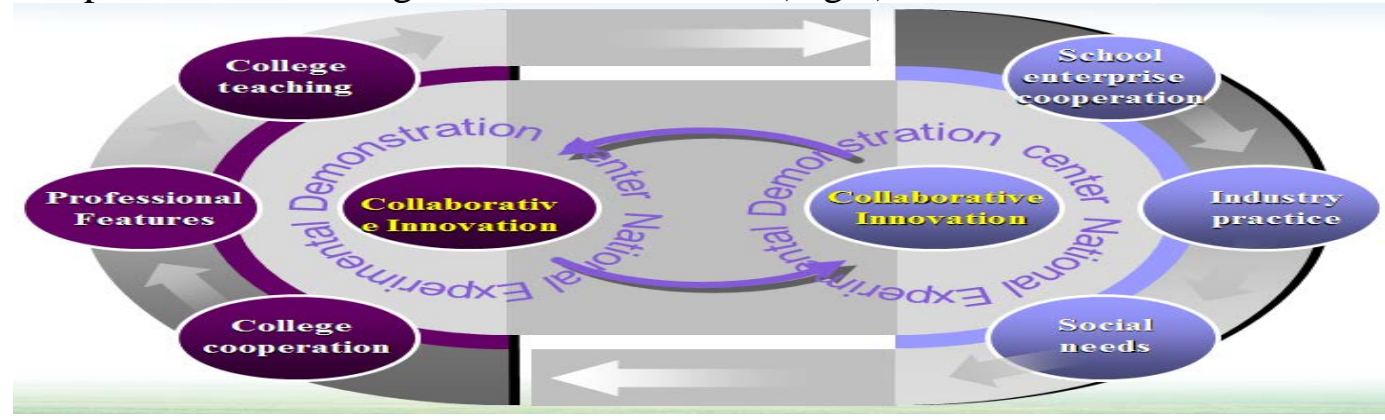

Figure 3 Internal and external collaborative innovation drives at the national experimental teaching demonstration center 


\section{Conclusion}

The national experimental teaching demonstration center has faced many difficulties. The 13th five-year plan clearly stipulates the strategy for sustainable development of demonstration centers, which points out the reform direction of experimental teaching. There, however, is still a long way to go to achieve the sustainable development. So the following suggestions are put. (1) A platform for cooperation with the industry should be built. Outstanding talents should be introduced into the experimental teaching classroom. (2) we advocate to conduct school-enterprise cooperation, which can provide more space for social practice, and which can reduce the time cost of adapting to society. (3) A "virtual combination" that breaks the single model of the traditional experimental teaching center should be built, which becomes the key to the sustainable development of experimental teaching demonstration center and stimulates the virtual experiment teaching demonstration center.

\section{References}

[1] Yang Ye, Wang Kebo, Li Yuan. 2017, Exploration of Teaching Reform of Experimental Teaching Demonstration Center in Colleges and Universities [J]. Education Teaching Forum, (02): 271-273.

[2] Pan Lei. 2014, Thoughts on the Sustainable Development of National Experimental Teaching Demonstration Center [J]. Experimental Technology and Management, 31(5):164-166.

[3] Sun Jianlin, Yan Bing, Jiang Wei. 2016, Experimental teaching demonstration center and virtual simulation experiment teaching center are mutually integrated and developed collaboratively [J]. Experimental Technology and Management, 09:208-214.

[4] [9] Lueking A D, Cole M W. 2017, Energy and mass balances related to climate change and emediation[J]. Science of the Total Environment, s 590-591:416-429.

[5] Lu Xiaoyan, Wen Yanxuan. 2016, Strategies of Cooperation Development between Local Universities and Enterprises in the Context of Collaborative Innovation [J]. Laboratory Research and Exploration, (9).

[6] Tong Yuerong. 2016, Exploration and reflection on the joint training of excellent engineering talents by schools and enterprises [J]. Chemical Industry in Higher Education, 33(1):13-17.

[7] [8] Chen Linyi, Shi Yan, Li Qinxi, Zhou Dawang, Zuo Zhenghong. 2018, Comprehensive Education Function of National Experimental Teaching Demonstration Center [J].Experimental Technology and Management, (06):222-225. 